\title{
Nierenzellkarzinom: Pazopanib mit günstigerem Verträglichkeitsprofil?
}

- Mit drei Tyrosinkinase-Inhibitoren (TKI), einem Antikörper gegen den vaskulären endothelialen Wachstumsfaktor (VEGF) und zwei mTOR-Inhibitoren stehen derzeit sechs Substanzen für die zielgerichtete Therapie des metastasierten Nierenzellkarzinoms (mRCC) zur Verfügung. Beim klarzelligen Karzinom mit niedrigem oder mittlerem Risiko empfehlen die aktuellen Leitlinien für die Erstlinientherapie neben Sunitinib und Bevacizumab auch Pazopanib (Votrient $^{\circledR}$ ). Der Multi-TKI ist seit Juni 2010 zugelassen.

Eine randomisierte doppelblinde placebokontrollierte Studie mit 435 Patienten mit mRCC hatte signifikante Vorteile für Pazopanib gezeigt, wie Prof. Christian Doehn,
Lübeck, berichtete [Sternberg et al. JCO 2010; 28: 1061-8]. Der TKI verlängerte das progressionsfreie Überleben (PFS) in der Gesamtgruppe von 4,2 auf 9,2 Monate, in der First-Line-Gruppe von 2,8 auf 11,1 Monate und in der Second-Line-Gruppe von 4,2 auf 7,4 Monate. Auch für die beiden anderen Substanzen zeigen die entsprechenden Phase-III-Daten eine Verdopplung des PFS, ein Ansprechen bei etwa einem Drittel der Patienten sowie ein um mindestens eineinhalb Jahre verlängertes Gesamtüberleben. Das gilt ähnlich auch für die Zweitlinientherapie, für welche die Leitlinien Sorafenib, Everolimus und Pazopanib empfehlen. Neben den Leitlinien und den Phase-IIIDaten müssen daher laut Doehn weitere
Faktoren für die Therapieentscheidung eine Rolle spielen - zum Beispiel die Toxizität. Direkte Vergleichsdaten fehlten zwar noch, beim Vergleich der Studien zeichne sich jedoch ein günstigeres Verträglichkeitsprofil bei Pazopanib ab, so Doehn. Grad 3/4-Nebenwirkungen, Fatigue und Blutungen, Stomatitis, Mukositis oder Hand-Fuß-Syndrom sind offenbar seltener als unter anderen TKI. Aufschluss erwartet Doehn hier von der aktuell laufenden COMPARZ-Studie, dem ersten direkten Vergleich mit Sunitinib bei nicht vorbehandelten Patienten.

koc

Satellitensymposium „Uro-Onkologie im Wandel" im Rahmen des 62. Kongresses der Deutschen Gesellschaft für Urologie,

Düsseldorf, 24. September 2010

Veranstalter: GlaxoSmithKline, München

\section{Erfolgreiche Prostatakarzinomtherapie mit GnRH-Antagonisten}

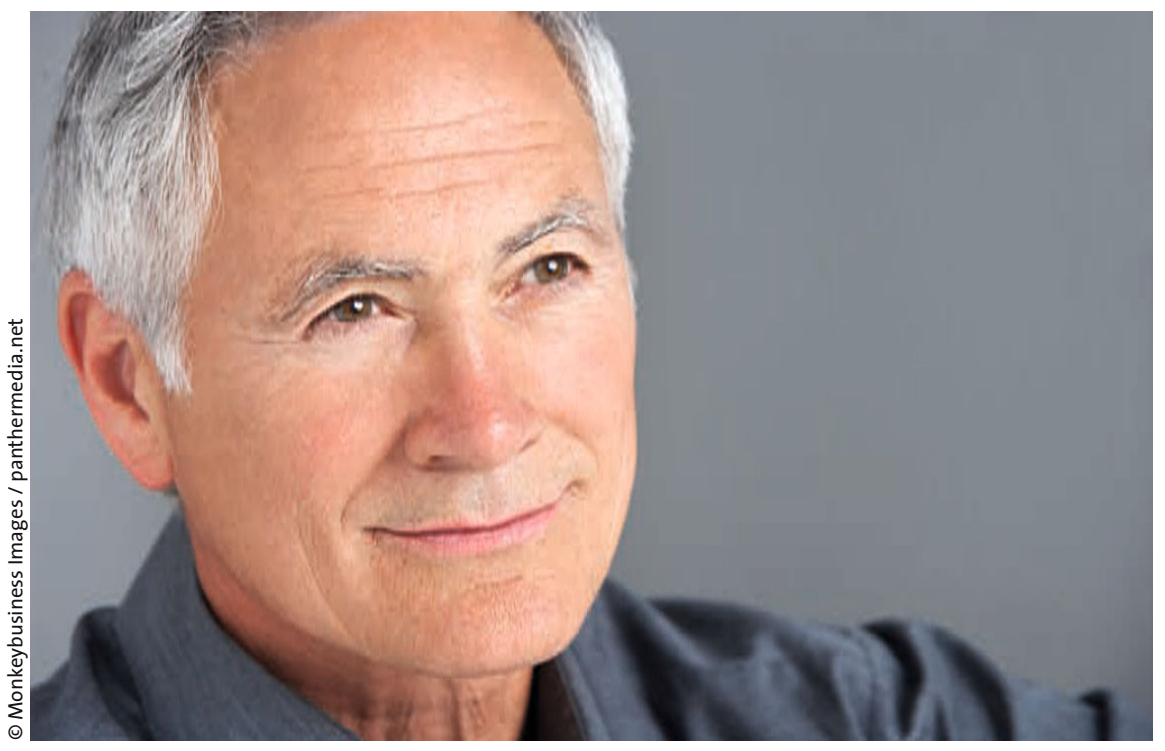

Hoffnung für Patienten mit Prostatakarzinom: Die Therapie macht Fortschritte.

- LHRH-Agonisten kommen seit den 8oerJahren als Ersatz für die Orchiektomie in der Behandlung des Prostatakarzinoms zum Einsatz. Allerdings kann laut Prof. Dr. Kurt Miller, Direktor der Urologischen Klinik der Charité, Berlin, mit den meisten dieser Substanzen der durch den chirurgischen Eingriff erzielte Testosteronwert von unter $0,2 \mathrm{ng} / \mathrm{ml}$ nicht erzielt werden. Seit etwa einem Jahr bieten GnRH-Antagonisten, auch GnRH-
Blocker genannt, wie beispielsweise Degarelix (Firmagon ${ }^{\circledR}$ ) eine Alternative zu den LHRH-Agonisten, um den Testosteronspiegel zu senken, so Miller.

Die theoretischen Vorteile von Degarelix gegenüber den LHRH-Agonisten sind laut Miller ein fehlendes Flare-up-Phänomen und fehlende Microsurges im Testosteronspiegel sowie eine schnelle und tiefe Testosteronabsenkung. In der CS21-Zulassungs- studie konnte gezeigt werden, dass der Wirkstoff in der Tat eine zuverlässige und schnelle Testosteronsenkung erzielen kann [Klotz LL et al. BJU Int 2008; 102: 1531-8). Im Rahmen der Phase-III-Studie wurde die Wirksamkeit des GnRH-Blockers (80 bzw. $160 \mathrm{mg}$ s. c. monatlich) mit dem LHRH-Agonisten Leuprorelin (7,5 mg i. m. monatlich) verglichen. Dabei zeigte sich, dass die Unterdrückung des PSA unter Degarelix signifikant schneller war. Bei Patienten, die Degarelix erhielten, konnte im Vergleich zu Patienten, die mit Leuprorelin behandelt wurden, zusätzlich ein signifikant längeres PSA-progressionsfreies Überleben festgestellt werden. Außerdem wurden unter Degarelix niedrigere Werte der alkalischen Phosphatase - einem Indikator für Knochenmetastasen - festgestellt als unter Leuprorelin. „Haben wir einen Fortschritt gemacht? - Ja! “ beurteilte Miller die Bedeutung dieser Ergebnisse abschließend.

Pressekonferenz „Testosteron - des einen Freud', des anderen Leid. Welche Rolle spielt Testosteron bei PCa und bei hypogonadalen Patienten?" im Rahmen des 62. Kongresses der Deutschen Gesellschaft für Urologie, Düsseldorf, 23. September 2010 Veranstalter: Ferring, Kiel 